\title{
Simulating Side Slopes on Locomotion Interfaces using Torso Forces
}

\author{
John M. Hollerbach \\ Univ. of Utah, School of Computing \\ 50 S. Central Campus Dr., Salt Lake City \\ UT 84112 USA \\ jmh@cs.utah.edu
}

\author{
Damaso Checcacci \\ PERCRO Lab, Scuola Superiore S. Anna \\ P.zza Martiri della Libertà 33, 56127 Pisa, Italy \\ checcacci@sssup.it
}

\author{
Haruo Noma, Yasuyuki Yanagida, Nobuji Tetsutani \\ ATR Media Information Sciences Laboratories \\ 2-2-2 Hikaridai, Keihanna Science City \\ Kyoto, 619-0288, Japan \\ \{noma,yanagida,tetsutani\}@atr.co.jp
}

\begin{abstract}
This paper describes the biomechanical experimental validation of simulating side slope during walking on a treadmill style locomotion interface. The side slope effect is achieved by means of a lateral force applied to the waist of the walking subject. Results are provided and discussed for both simulated and real side slopes, showing a substantial biomechanical equivalence in the walking pattern for the real side slope and lateral torso force.
\end{abstract}

\section{Introduction}

A key issue for locomotion interfaces is the presentation of sloping terrain. One approach is programmable foot platforms, which can simulate adjustable stair-like terrain, as in the Sarcos Biport [6] or Iwata's Gaitmaster [7]. A common approach for treadmill style locomotion interfaces is an up-down tilting platform. In addition, sideways tilt can be added to present sideslope walking as in the ATR ATLAS [11].

Instead of tilting a treadmill, which increases mechanical complexity and complicates the use of CAVE-like visual displays, another approach is to apply forces to the torso of a user. It was shown in [4] that horizontal forces in the forward direction applied by the active mechanical tether in the Sarcos Treadport locomotion interface yield realistic simulation of uphill slope walking, both from psychological and biomechanical standpoints. Other demonstrated advantages of torso force feedback are collision simulation and inertial force simulation [1].
A natural follow-on question is whether applying side forces to a subject walking on a level treadmill could produce an effect similar to walking on a real side slope. If so, then there may not be any need to move the treadmill platform at all. The required actuator forces for application to the torso are much less than for tilting a platform, especially a large platform such as in the Sarcos Treadport. The bandwidth would also be higher, allowing faster slope transients to be displayed. The vision of future treadmill-style locomotion interfaces would therefore consist of a stationary platform, perhaps with some form of two-dimensional surface $[2,8]$, with terrain and other effects provided by torso force feedback.

This paper performs a biomechanical comparison of walking on a treadmill tilted sideways versus walking on a horizontal treadmill with passive torso forces. The ATLAS system was employed throughout.

\section{Methods}

The ATLAS locomotion interface [11] has a treadmill platform mounted on an active spherical joint. It can rotate around a vertical axis and tilt both sideways and longitudinally, in order to reproduce a general ground orientation. In the present study, its sideways tilting capability was essential. Side forces were applied by a simple pulley/weight system. An active tether for side forces is not available yet; the decision to build one hinges upon the successful outcome of these feasibility studies.

Eleven subjects ( 8 males, 3 females, aging $20 \pm 3$ years) were asked to walk on the ATR treadmill both in a real side slope condition and under the application of a lateral force. 


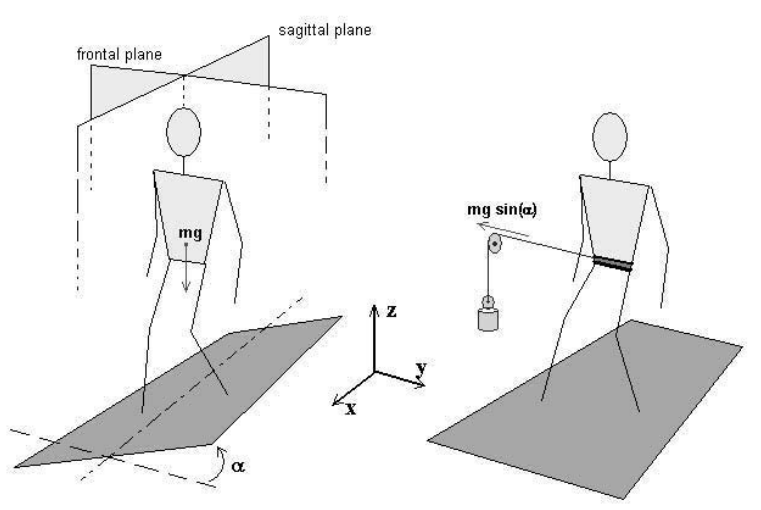

Figure 1. Layout of the experiment with indication of the reference planes.

The real slope was varied in the range $\left[-16^{\circ},+16^{\circ}\right]$ with increments of $2^{\circ}$. The force was applied using weights that were connected to the subject's waist belt by a cable/pulley apparatus. Several weights were successively added to provide a force in the range $[-120,+120] \mathrm{N}$, with increments of $15 \mathrm{~N}$.

Two walking speeds were employed under each condition, $3 \mathrm{~km} / \mathrm{h}$ and $5 \mathrm{~km} / \mathrm{h}$. The treadmill speed was ramped up and down at a rate of $1 \mathrm{~km} / \mathrm{h} / \mathrm{sec}$. Once the desired treadmill speed had been attained, enough data was collected for roughly 5 step cycles, then the speed was ramped down.

Considering a coordinate system with the $\mathbf{z}$ and $\mathbf{x}$ axes directed upward and in the forward direction of walking respectively (Figure 1), then a positive side slope angle means a positive rotation around the $\mathbf{x}$ axis. A positive force, instead, is intended in the negative direction of the $\mathbf{y}$ axis. In Figure 1 both force and slope are reported in their positive direction. With this convention we expect a positive force to be required to simulate a positive slope and vice versa.

To track the walking motion of the subjects, the QuickMAG IV by OKK Inc., Japan, was used. The system employs two cameras and colored markers, which are sampled at $60 \mathrm{~Hz}$ with an accuracy of $1.5 \mathrm{~mm}$ in the side and uphill directions, and $2.5 \mathrm{~mm}$ in depth. Five markers were used to track points located on the torso, hip, knee, ankle and foot tip, on the left side of the body. In Figure 2, the marker positions are shown as well as the side force apparatus. Two additional markers were used to define a plumbline in order to determine the vertical reference for data postprocessing. Calibration data were collected for the axes orientation (plumbline and treadmill direction) and the zero positions of the markers during static standing, for every subject.

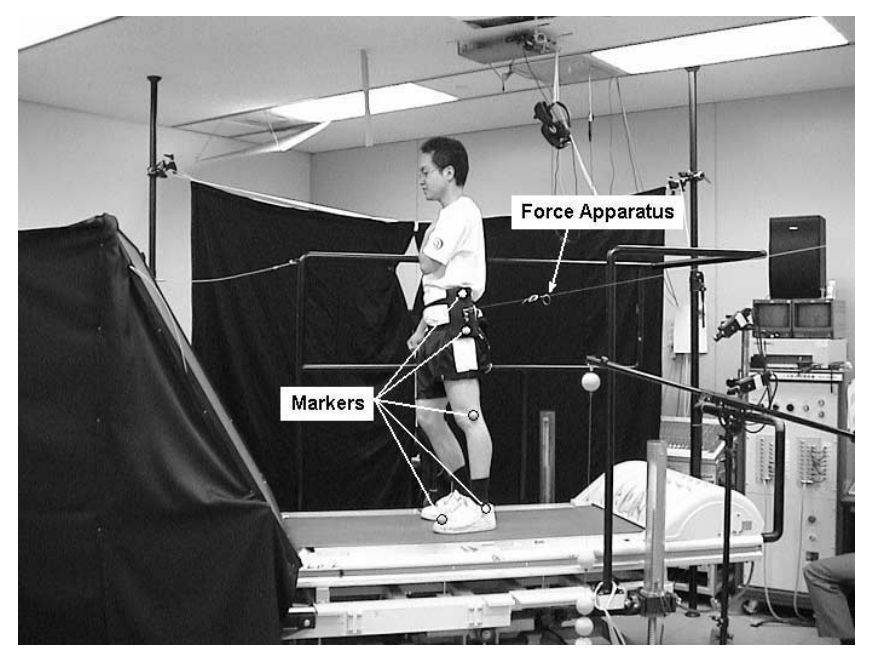

Figure 2. The experimental setup on the ATLAS system.

\section{Experimental Results}

The aims of this study were (1) an investigation of the possibility to realistically simulate a side slope by means of an externally applied force, and (2), in the case of a positive result, the establishment of a force-slope analytical relation that could be used in order to actually implement such a simulation in a locomotion interface.

To compare the gait data for the real and simulated walking, the following angles have been computed starting from the markers' $3 \mathrm{D}$ positions:

- the upper body angle in both sagittal and frontal planes;

- the hip, knee and ankle angles, that are typically used to parameterize 2D gait in the sagittal plane [3];

- the thigh and ankle abduction angles (formed with the sagittal plane). The last is projected onto the frontal plane.

In Figure 1 a layout of the experimental procedure and the above mentioned reference planes are sketched, while Figure 3 shows the angles in both the sagittal and frontal planes. The abduction angles and the upper body orientation in the frontal plane seemed particularly useful for analyzing the effects of side slope and force. Indeed no particular effect is expected in the sagittal plane since all the perturbations (both geometric and static) occur in the frontal plane.

Figures 8 and 9 show the results (mean angles values) over all subjectss and all applied slopes and forces, at a 3 

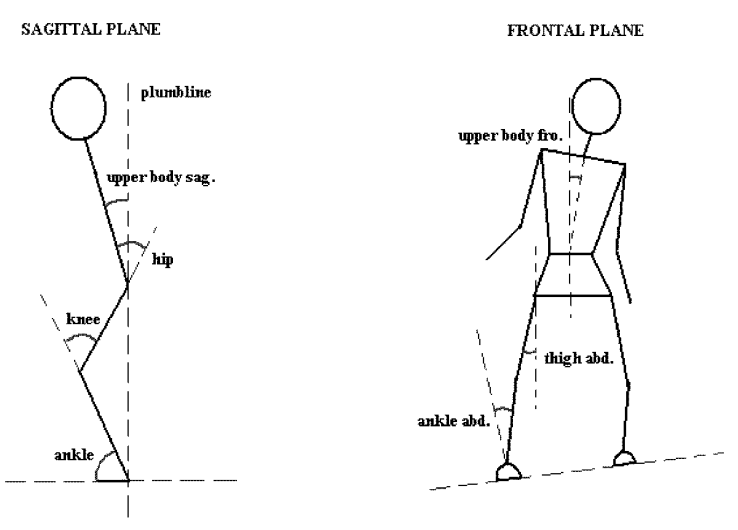

Figure 3. Reference angles sketch.

$\mathrm{km} / \mathrm{h}$ walking speed. The corresponding ranges are reported in Figures 10 and 11.

The plots refer to the tracking of the subject's left side only, but the data have been collected over symmetric intervals. Opposite points (i.e. slope $-16^{\circ}$ and $+16^{\circ}$ ) can be regarded as data collected from the two opposite legs in the same walking conditions.

From previous experiments on uphill slopes [5], it was determined that one of the most linear features with slope is the hip range, but from Figure 10 it is clear that this relation no longer holds for side slope walking. Instead, from Figure 9 it can be seen that the only feature that varies consistently with the side slope is the ankle abduction mean angle. Thus, on side slope walking, the subjects basically maintain the same walking posture and simply change the ankle abduction angle in order to adapt the foot to the current floor inclination. Indeed the thigh abduction mean angle is unchanged, thus showing that the legs continue to lie on the same plane as in level walking.

By similar considerations for the case of side force walking, it can be seen that, again, the ankle abduction mean angle varies consistently with slope, thus providing a reliable equivalence mean between the real and the simulated side slope.

Nevertheless, by applying a side force this is not the only effect that is achieved. The thigh mean angle changes as the subject widens the legs in order to provide a more stable support to contrast the lateral force, see Figure 9. This means that the relative position of the legs with respect to the treadmill platform is the same, but, since the platform is now horizontal, the absolute orientation of the subject's legs with respect to the vertical reference must have changed.

The other features being basically equivalent, both in real slope and in the simulated one, leads to the conclusion that the gait of the subjects, in the two different conditions, were practically the same, thus providing a biomechanical proof

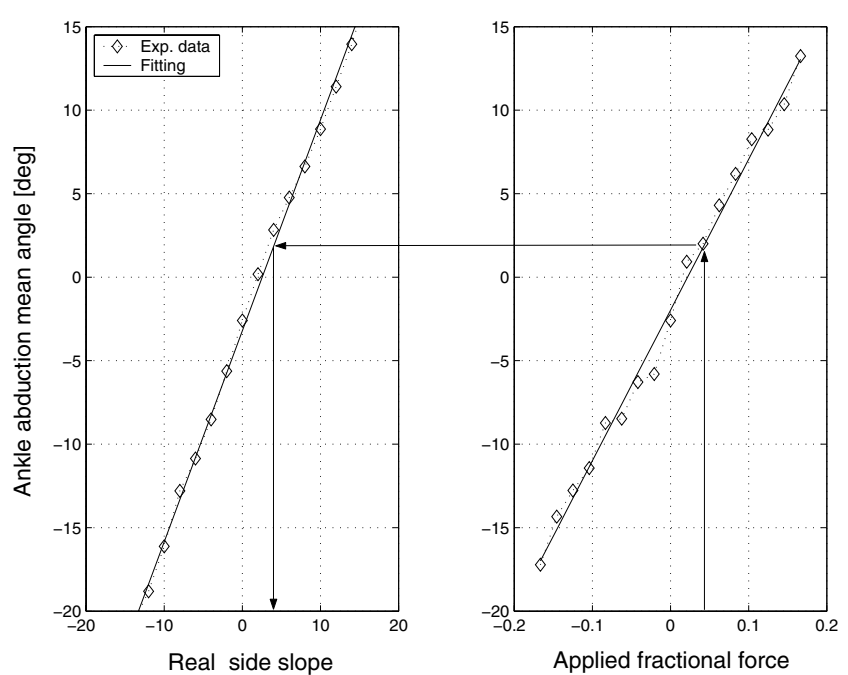

Figure 4 . Equivalence of applied fractional
force to real slope, for one subject, on the
basis of ankle abduction mean angle.

of the simulation method validity.

Moreover a good linear relation seems to hold between the ankle abduction mean angle and both the side slope and the applied force, thus providing a good basis to find a linear relation between the simulated slope and the applied weight. This is carried on in the following section.

\section{Relation between Simulated Side Slope and Lateral Force}

To compare results across subjects, it is necessary to normalize the lateral forces $W$ according to each subject's mass $m$, which ranged from 46.5 to $75.5 \mathrm{~kg}$. The ratio of lateral force to body weight $m g$ is termed the applied fractional force $\mathcal{F}=W / m g$. By comparing the plots of ankle abduction angle versus side slope $\theta$ and versus applied fractional force, a relation could be determined between side slope and applied lateral force.

- For every subject, the data of side force versus ankle abduction angle was replotted in terms of applied fractional force (right side of Figure 4).

- A straight line was fit between the ankle abduction angle and the real slope (left side of Figure 4).

- An applied fractional force was correlated to a side slope by matching the ankle abduction angles using the straight-line fit above. 
Table 1. Summary of interpolation results over individual subjects. Speed $=3 \mathrm{~km} / \mathrm{h}$

\begin{tabular}{c|ccccc}
\hline Subject & $k_{1}$ & $k_{0}$ & Stnd.D. & Corr. C. & $\theta_{W=0}$ \\
\hline 1 & 0.922 & 0.002 & 0.011 & 0.997 & -0.123 \\
2 & 0.985 & 0.038 & 0.030 & 0.977 & -2.187 \\
3 & 1.242 & 0.000 & 0.039 & 0.938 & -0.012 \\
4 & 0.798 & -0.014 & 0.009 & 0.996 & 0.978 \\
5 & 0.853 & -0.001 & 0.007 & 0.999 & 0.091 \\
6 & 0.900 & 0.001 & 0.013 & 0.993 & -0.079 \\
7 & 0.900 & -0.027 & 0.011 & 0.996 & 1.724 \\
8 & 0.811 & -0.032 & 0.012 & 0.994 & 2.237 \\
9 & 0.768 & 0.018 & 0.022 & 0.986 & -1.319 \\
10 & 1.001 & -0.051 & 0.020 & 0.989 & 2.902 \\
11 & 0.839 & -0.023 & 0.011 & 0.997 & 1.562 \\
\hline
\end{tabular}

Table 2. Summary of interpolation results over individual subjects. Speed $=5 \mathrm{~km} / \mathrm{h}$

\begin{tabular}{c|ccccc}
\hline Subject & $k_{1}$ & $k_{0}$ & Std. Dev. & Corr. C. & $\theta_{W=0}$ \\
\hline 1 & 0.899 & 0.000 & 0.013 & 0.995 & -0.013 \\
2 & 0.937 & 0.037 & 0.037 & 0.965 & -2.284 \\
3 & 1.156 & -0.003 & 0.030 & 0.964 & 0.129 \\
4 & 0.705 & -0.013 & 0.007 & 0.998 & 1.089 \\
5 & 0.805 & -0.003 & 0.013 & 0.997 & 0.238 \\
6 & 0.766 & -0.002 & 0.013 & 0.992 & 0.113 \\
7 & 0.904 & -0.031 & 0.011 & 0.996 & 1.970 \\
8 & 0.854 & -0.030 & 0.008 & 0.997 & 2.037 \\
9 & 0.785 & 0.017 & 0.014 & 0.994 & -1.251 \\
10 & 0.871 & -0.027 & 0.014 & 0.994 & 1.800 \\
11 & 0.768 & -0.007 & 0.015 & 0.995 & 0.551 \\
\hline
\end{tabular}

A simple theoretical model predicts a relation $W / m g=$ $\tan \theta$, but because the data do not exactly fit this theoretical model a more general straight-line equation was fit:

$$
\frac{W}{m g}=k_{1} \tan \theta+k_{0} \approx k_{1} \theta+k_{0}
$$

where the approximation $\tan \theta \approx \theta$ has a maximum error of only $2.6 \%$ at the slope of $16^{\circ}$. The individual interpolation results are summarized in Tables 1-2.

For the $3 \mathrm{~km} / \mathrm{h}$ walking speed, the means of the results for all subjects is:

$$
k_{1_{3 k m / h}}=0.911 \quad k_{0_{3 k m / h}}=-0.008
$$

with mean standard deviation of error of 0.016 and correlation coefficient of 0.987 . The fact that the intercept $k_{0}$ is not zero is due to experimental errors. For a subject of $70 \mathrm{~kg}$, this means an error of about $5.5 \mathrm{~N}$ at zero slope (or

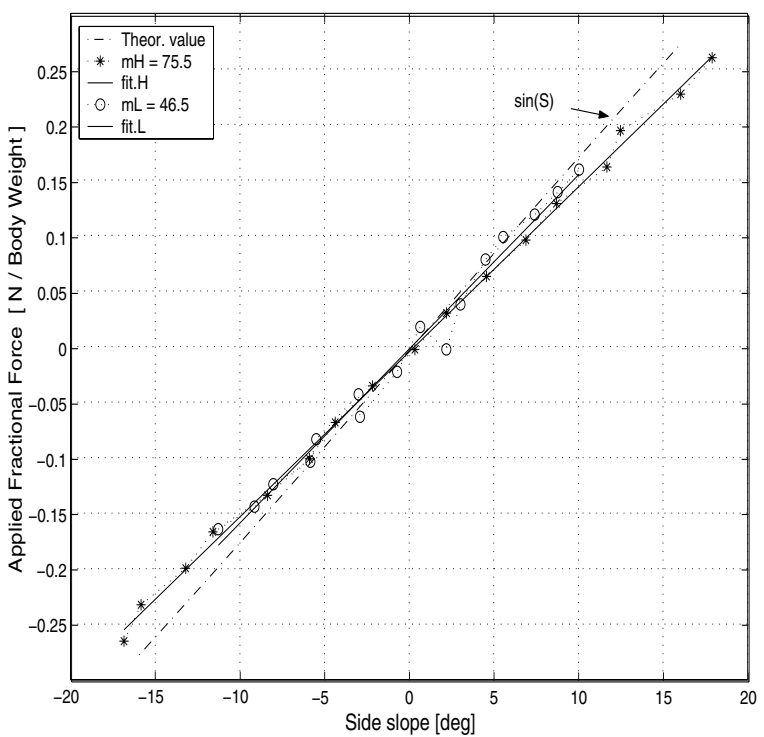

Figure 5. Interpolation of fractional force versus real perceived slope. Raw experimental points and fittings are shown for the lightest and heaviest subject. Speed $=3 \mathrm{~km} / \mathrm{h}$.

$\theta_{0}=-k_{0} / k_{1}[\mathrm{rad}]=0.5^{\circ}$ in terms of slope at zero force for the inverse relation). In Figure 5 the line of the theoretical relation $W / m g=\tan (\theta)$ is also given.

For the $5 \mathrm{~km} / \mathrm{h}$ walking speed, there are no substantial changes but a slight general increase of the ranges over all measured angles. In this case the fitting over all subjects provides the following mean results:

$$
k_{1_{5 k m / h}}=0.859 \quad k_{0_{5 k m / h}}=-0.0057
$$

with mean standard deviation of error of 0.015 , correlation coefficient of 0.989 , and a slope error at zero force of $0.37^{\circ}$.

In Figure 5 the raw data and the interpolation for the lightest and heaviest subject are compared for the $3 \mathrm{~km} / \mathrm{h}$ walking speed. Since the lateral forces were the same over all subjects, the fractional force varies over a wider range for a light subject than for a heavy one. As a consequence there are more data points for small values of slopes and fractional forces than for large ones.

The similarity shows that normalization based upon a subject's weight has identified a general trend across subjects. While the relation between side slope and applied fractional force is strongly linear for all subjects, there is a significant variation in slope for different subjects, \pm 0.2 . Since the mean coefficients $k_{1_{3 \mathrm{~km} / h}}$ and $k_{1_{5 \mathrm{~km} / h}}$ are also very similar to each other, the general trend seems to hold across walking speeds. 


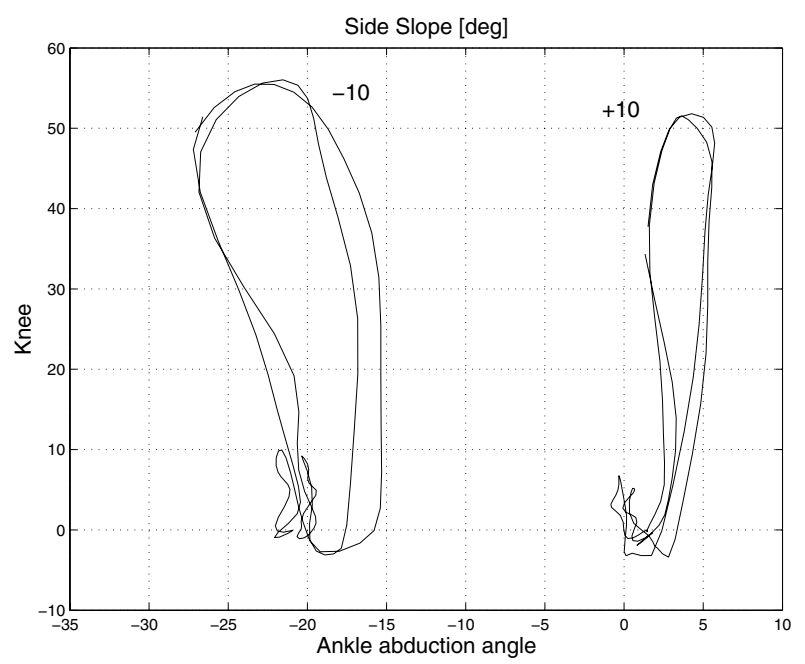
Figure 6. Ankle abduction/knee cyclograms
for a single subject walking on a real slope of
$\pm 10^{\circ}$. Speed $=\mathbf{5} \mathrm{km} / \mathrm{h}$

That the $k_{1}$ coefficients are less than 1 means that a smaller lateral force than predicted by the simple model $W=m g \tan \theta$ is required, roughly $90 \%$. This is consistent with previous tether force studies: $80 \%$ of predicted force for inertial force display [1] and $65 \%$ of predicted force for uphill force display [5].

\section{Discussion}

In this paper a first result for the understanding and implementation of side slope simulation on treadmill style locomotion interfaces has been presented. The existence of a linear relation, between the mean abduction ankle angle and both the real side slope and the lateral applied fractional force, has been proven by experimental results. Examples of cyclograms for ankle abduction versus knee angle are reported in Figures 6 and 7 for both the real and simulated slope. The cyclograms show a close similarity both for shape and position.

The ankle abduction angle has been used as a biomechanical basis of equivalence between side slope and lateral force. The results demonstrate that a side slope $\theta$ can be simulated by application of a lateral force $W=$ $k_{1} m g \theta$. The proportionality coefficient $k_{1}$ is around 0.9 ( 0.88 with standard deviation of 0.12 ), and shows some variation across subjects and walking speed. A more precise and reliable proportional value could be defined by further experiments over a larger number of subjects. Since only two walking speeds, $3 \mathrm{~km} / \mathrm{h}$ and $5 \mathrm{~km} / \mathrm{h}$, were employed, a more exact relation with respect to walking speed cannot

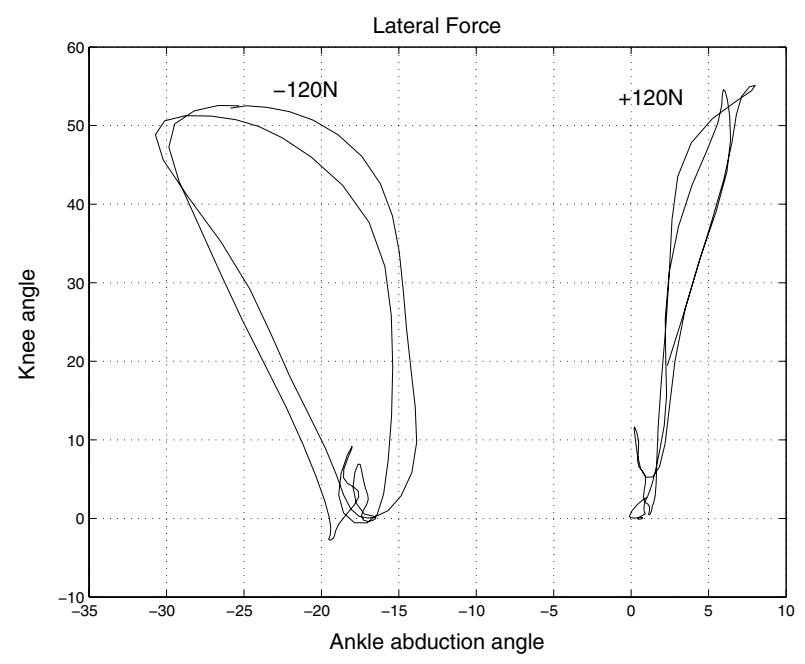

Figure 7. Ankle abduction/knee cyclograms for a single subject walking with an applied lateral force of $\pm 120 \mathrm{~N}$. Speed $=\mathbf{5} \mathbf{~ k m} / \mathbf{h}$

be reliably established yet. Nevertheless, a value of roughly $90 \%$ of the theoretical lateral force is probably good enough for locomotion display purposes without the need for tuning for each individual.

That this proportionality coefficient is less than 1 is not surprising, since in previous studies a correction factor of about 0.65 had already been established for uphill slopes [4] and 0.8 for inertial forces [1]. A thorough investigation for the reason for these discrepancies awaits further analysis. Nevertheless, a first explanation on the difference between the fractional force that is required in the sagittal and frontal planes (simulation of uphill and side slope respectively) can be intuitively found by energetic considerations.

- In uphill walking, the slope acts in the walking direction, requiring a net work for subjects to advance and increase their potential energy (even if this does not hold in tilted treadmill walking). So the main goal of the simulation force is matching the energy cost of the real uphill walking.

- In the side slope experiments, instead, the slope acts in a direction that is orthogonal to that of walking. So, theoretically, no different power is required to the user to advance. The lateral static balance is the main factor affecting the choice of the simulation force.

An interesting test would be that of performing the complimentary experiments of side walking on a side slope (energy involved) and side walking on a uphill slope (balance involved). 
However the biomechanics of the human body itself must be taken into account. The human body, indeed, is particularly suited to control the balance in the sagittal plane (that is the main plane of swing during normal walking). Consistent lateral perturbations, instead, are much more uncommon to us, and balancing against side forces is more difficult [9]. Indeed, in the performed experiments, the subjects showed a particular difficulty in balancing themselves while walking with an applied lateral force. This resulted also in a slight increase of the frontal angles ranges in the case of lateral force with respect to side slope (last three graphs of Figures 10 and 11), as well as a general higher standard deviation across subjects. This difficulty was higher at the lower speed, when the natural step-bystep balance correction process occurs at a lower rate.

The results of this paper further support the notion that general slopes can be displayed without tilting the treadmill platform. This can simplify the locomotion interface design while providing additional benefits from torso force feedback, such as inertial force display and collision forces. The results therefore support the next step of building an active force display in the side direction, adding to the existing force display of the Treadport in the frontal direction. Such planar torso force feedback would be useful for any treadmill-style locomotion interface, including twodimensional belt surfaces $[2,8]$. The active side force could also assist users to balance by a combination of position and force control. More complete body tracking would probably be required to infer a subject's lean.

\section{Acknowledgments}

This research was supported by NSF Grants ITR/SY(IIS)-0113996 and ITR/SY-0121084, and by the Telecommunications Advancement Organization of Japan. We acknowledge the technical assistance of Mamoru Saijyo.

\section{References}

[1] Christensen, R.R., Hollerbach J.M., Xu, Y., and Meek, S.G. 2000. Inertial-force feedback for the Treadport locomotion interface. Presence: Teleoperators and Virtual Environments. 9: 1-14.

[2] Darken, R.P., and Cockayne, W.R. 1997. The OmniDirectional Treadmill: a locomotion device for virtual worlds. Proc. UIST. pp. 213-221.

[3] Goswami, A. 1998. A new gait parameterization technique by means of cyclogram moments: application to human slope walking. Gait \& Posture. 8:15-36
[4] Hollerbach, J.M., Mills, R., Tristano, D., Christensen, R.R., Thompson, W.B. and Xu, Y. 2001. "Torso force feedback realistically simulates slope on treadmillstyle locomotion interfaces." Intl. J. Robotics Research. 20:939-952.

[5] Hollerbach, J.M., Xu, Y., Christensen, R., and Jacobsen, S.C. "Design specifications for the second generation Sarcos Treadport locomotion interface" Haptic Symposium, Proceedings of ASME Dynamic Systems and Control Division, DSC-Vol.69-2, Orlando, FL, November 2000, pp. 1293-1298.

[6] Hollerbach, J.M., "Locomotion interfaces," in: Handbook of Virtual Environments Technology, K.M. Stanney, ed., Lawrence Erlbaum Associates, Inc., 2002, pp. 239-254.

[7] Iwata, H. 2000. Locomotion interface for virtual environments. Robotics Research: The Ninth International Symposium. pp. 275-282.

[8] Iwata, H., and Yoshida, Y. 1999. Path reproduction tests using a Torus Treadmill Presence: Teleoperators and Virtual Environments. 8:587-597.

[9] Leroux, A., Fung, J., Barbeau, H. 2002. Postural adaptation to walking on inclined surfaces: I. Normal strategies. Gait \& Posture, Vol. 15, pp.:64-74.

[10] Noma, H., Sugihara,T., and Miyasato, T. “Development of ground surface simulator for tel-E-merge system". Preceedings of IEEE Virtual Reality 2000,New Brunswich, NJ, March 2000, pp.217-224.

[11] Noma, H., and Miyasato, T. "Design for locomotion interface in a large scale virtual environment. ATLAS:ATR Locomotion Interface for Active Self Motion". Preceedings of ASME Dynamic Systems and Control Division, DSC-Vol. 64, 1998, pp.111-118. 

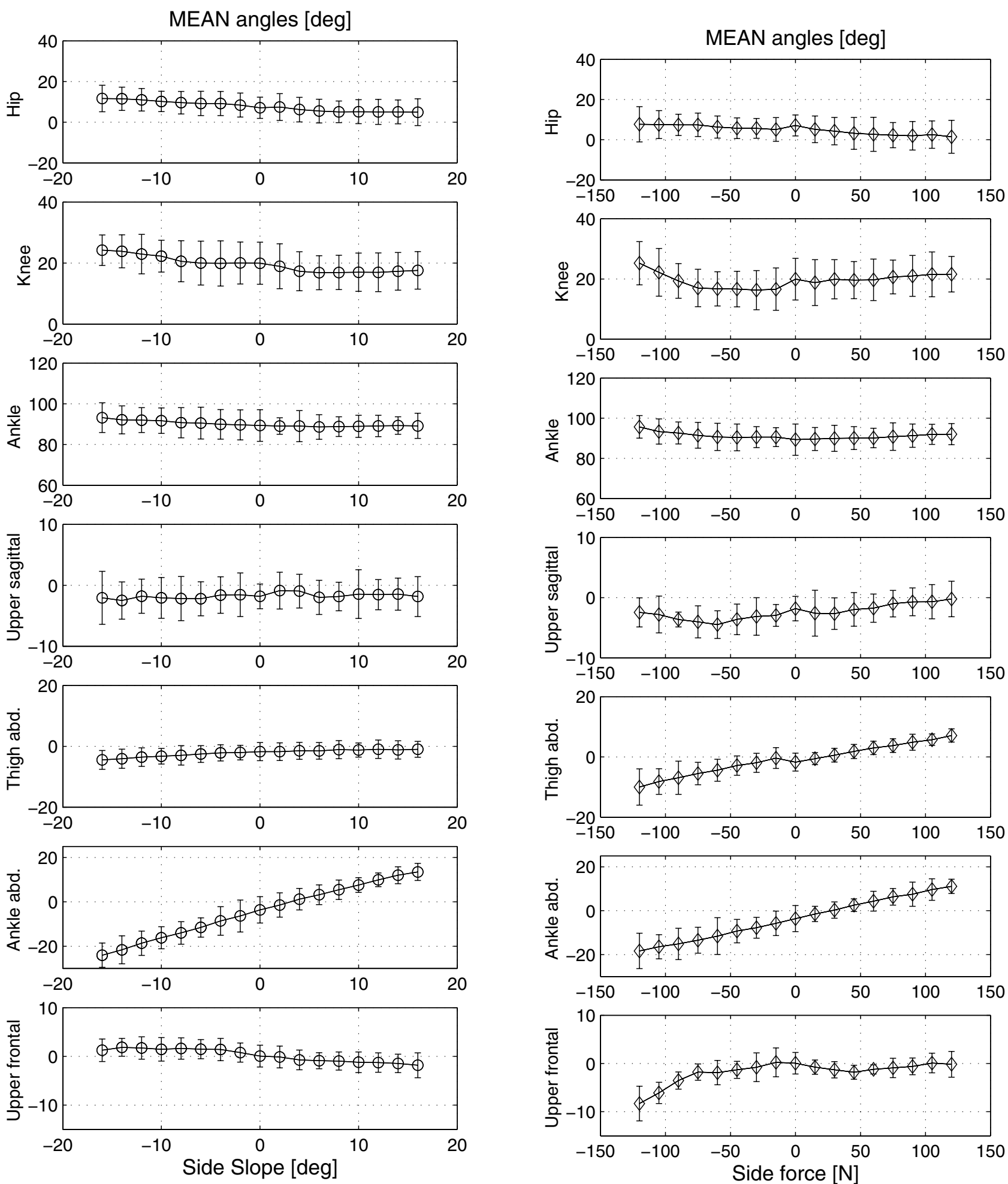

Figure 8. Mean angles over all subjects walking on a real side slope. Standard deviation across subjects is aslo shown.

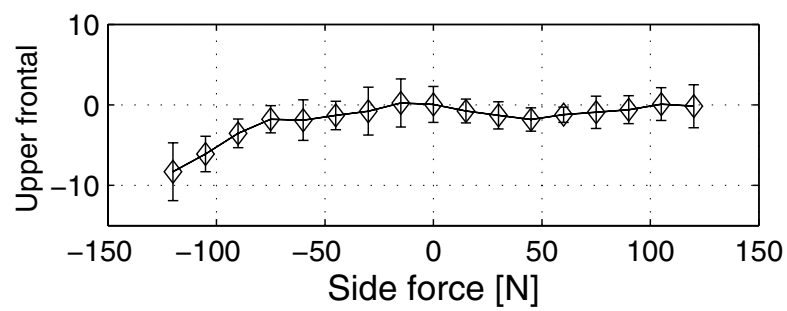

Figure 9. Mean angles over all subjects walking with an applied side force. 

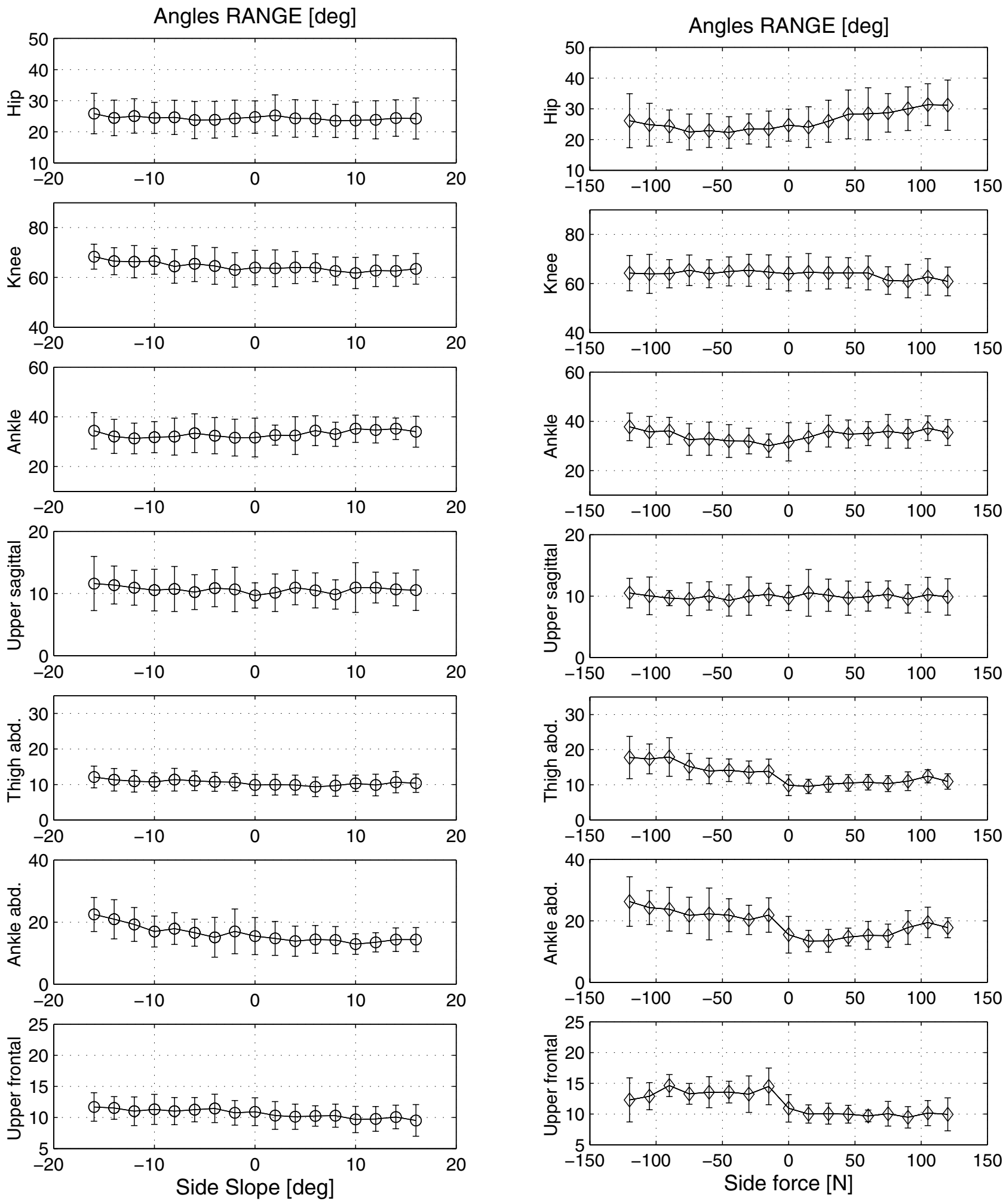

Figure 10. Measured ranges over all subjects walking on a real side slope. Standard deviation across subjects is aslo shown.

Figure 11. Measured ranges over all subjects walking with an applied side force. 